\title{
A High Resolution Search for Dark-Matter Axions
}

\author{
L. D. Duffy, P. Sikivie * $^{2}$ and D. B. Tanner \\ Department of Physics, University of Florida, Gainesville, Florida 32611, USA
}

\author{
S. J. Asztalos, C. Hagmann, D. Kinion, L. J Rosenberg, K. van Bibber, and D. B. Yu \\ Lawrence Livermore National Laboratory, Livermore, California 94550, USA \\ R. F. Bradley \\ National Radio Astronomy Observatory, Charlottesville, Virginia 22903, USA
}

(Dated: March 3, 2006)

\begin{abstract}
We have performed a high resolution search for galactic halo axions in cold flows using a microwave cavity detector. The analysis procedure and other details of this search are described. No axion signal was found in the mass range 1.98-2.17 $\mu \mathrm{eV}$. We place upper limits on the density of axions in local discrete flows based on this result.

PACS numbers: 14.80.Mz, 95.35.+d, 98.35.Gi
\end{abstract}

\section{INTRODUCTION}

In the current concordance cosmology, $23 \%$ of the universe's total energy density is contributed by exotic dark matter [1]. The axion, arising from the Peccei-Quinn solution to the strong CP problem [2], satisfies the two criteria necessary for cold dark matter [3]: (1) a very cold population of axions could be present in our universe in sufficient quantities to provide the required dark matter energy density and (2) axions are effectively collisionless; i.e., their only significant long-range interaction is gravitational. The Axion Dark Matter eXperiment (ADMX) [4] uses a Sikivie microwave cavity detector [5] to search for axions in our galactic halo.

The power in an axion signal observed by a microwave cavity detector is proportional to the local axion density. The signal width is caused by the velocity dispersion of dark-matter axions. Therefore, in searching for axions it is necessary to make some assumptions about their velocity distribution in our galactic halo. A variety of galactic halo models have been put forward: the isothermal model, results from N-body simulations $[\underline{6}]$ and the caustic ring model 7,8$]$. The predictions of these models are used to guide ADMX's search.

In the isothermal model it is expected that a significant fraction of the dark matter halo will have an isothermal velocity distribution resulting from a period of "violent relaxation" of the early galaxy [9]. This component of the halo will have velocities described by a MaxwellBoltzmann distribution. The "medium resolution" (MR) channel [10] searches for these axions, assuming that

\footnotetext{
*On leave during Fall 2005 at School of Natural Sciences, Institute for Advanced Study, Princeton, New Jersey 08540, USA, and during Spring 2006 at Theoretical Physics Division, CERN, CH1211 Genève 23, Switzerland

${ }^{\dagger}$ Present address: Piper Jaffray, LLC, 245 Park Ave., 33rd floor, New York, New York 10167, USA
}

the velocity dispersion is $\mathcal{O}\left(10^{-3} c\right)$ or less. (The escape velocity from our galaxy for axions is approximately $2 \times 10^{-3}$ c.)

N-body simulations and the caustic ring model both predict substructure within halos. Numerical simulations indicate that hundreds of smaller clumps, or subhalos, exist within the larger halo [6]. Tidal disruption of these subhalos leads to flows in the form of "tidal tails" or "streams". The Earth may currently be in a stream of dark matter from the Sagittarius A dwarf galaxy [1].

Non-thermalized flows from late infall of dark matter onto the halo are also expected [12]. Insufficient time has elapsed for dark matter that has fallen into the gravitational potential of the galaxy after violent relaxation to thermalize with the rest of the halo. Matter which has fallen onto the galaxy only recently will be present in the halo in the form of discrete flows. There will be one flow of particles falling into the gravitational potential for the first time, one flow of particles falling out for the first time, one due to particles falling in for the second time, etc. Furthermore, where the gradient of the particle velocity diverges, particles "pile up" and form caustics. In the limit of zero flow velocity dispersion, caustics have infinite particle density. The velocity dispersion of cold axions at a time, $t$, prior to galaxy formation is approximately $\delta v_{a} \sim 3 \times 10^{-17}\left(10^{-5} \mathrm{eV} / m_{a}\right)\left(t_{0} / t\right)^{2 / 3}[\underline{8}$, where $t_{0}$ is the present age of the universe and $m_{a}$ is the axion mass, constrained to lie between $10^{-6}$ and $10^{-2} \mathrm{eV}$ by cosmology and astrophysical processes 13]. Thus, a flow of dark matter axions will have a small velocity dispersion, leading to large, but finite density at the location of a caustic.

The caustic ring model predicts that the Earth is located near a caustic feature [14]. Fitting the model to bumps in the Milky Way rotation curve and a triangular feature seen in the IRAS maps predicts that the flows falling in and out of the halo for the fifth time contain a significant fraction of the local halo density. The predicted densities are $1.7 \times 10^{-24} \mathrm{~g} / \mathrm{cm}^{3}$ and $1.5 \times 10^{-25} \mathrm{~g} / \mathrm{cm}^{3}$ [14], comparable to the local dark 
matter density of $9.2 \times 10^{-25} \mathrm{~g} / \mathrm{cm}^{3}$ predicted in [15]. The flow of the greatest density is referred to as the "Big Flow". The possible existence of discrete flows, or streams, provides an opportunity to increase the ADMX discovery potential. A discrete axion flow produces a narrow peak in the spectrum of microwave photons in the experiment and such a peak can be searched for with higher signal-to-noise than a signal from axions in an isothermal model halo. The "high resolution" (HR) channel was built to take advantage of this opportunity. Furthermore, if a signal is found, the HR channel will provide us with detailed information on the structure of the Milky Way halo.

The HR channel is the most recent addition to ADMX, implemented as a simple addition to the receiver chain, running in parallel with the MR channel. This channel and the possible existence of discrete flows can improve ADMX's sensitivity by a factor of 3 [16], significantly enhancing its discovery potential. The full ADMX detector is described in Section II Each discrete flow of cold axions with small velocity dispersion will be seen as a narrow peak in the detector's output spectrum. Our expectations for a signal are discussed in Section III which also contains the details of the HR analysis, the primary topic of this paper. After a full search of the frequency range 478-525 MHz, no axion signals were found and we place limits on the density of cold axions in discrete flows in Section IV] This limit is compared to our previous results for the MR channel and halo substructure predictions in Section $\mathrm{V}$

\section{AXION DARK MATTER EXPERIMENT}

ADMX uses a microwave cavity detector to search for axions in our galactic halo. We outline the principle of the detector and briefly describe ADMX. Further details of the experiment can be found in [4, 19].

The microwave cavity detector utilizes the axionelectromagnetic coupling to induce resonant conversion of axions to photons. The relevant interaction is

$$
\mathcal{L}_{a \gamma \gamma}=g_{\gamma} \frac{\alpha}{\pi} \frac{a(x)}{f_{a}} \mathbf{E} \cdot \mathbf{B},
$$

where $\mathbf{E}$ and $\mathbf{B}$ are the electric and magnetic fields, $\alpha$ is the fine structure constant, $f_{a}$ is the axion decay constant, $a(x)$ is the axion field and $g_{\gamma}$ is a modeldependent coupling, of order one. In the Kim-ShifmanVainshtein-Zakharov (KSVZ) model [17], $g_{\gamma}=-0.97$, and in the Dine-Fischler-Srednicki-Zhitnitsky (DFSZ) model [18], $g_{\gamma}=0.36$. Axions in the galactic halo are non-relativistic, i.e. the energy of a single axion with mass $m_{a}$ and velocity $v$ is

$$
E_{a}=m_{a} c^{2}+\frac{1}{2} m_{a} v^{2},
$$

where $c$ is the speed of light. The axion-to-photon conversion process conserves energy, i.e. an axion of energy
$E_{a}$ converts to a photon of frequency $\nu=E_{a} / h$. When $\nu$ falls within the bandwidth of a cavity mode, the conversion process is resonantly enhanced. The signal is a peak in the spectrum output by the detector. The power, $P$, developed in the cavity due to resonant axion-photon conversion is [5]

$$
P=\left(\frac{\alpha g_{\gamma}}{\pi f_{a}}\right)^{2} \frac{V B_{0}^{2} \rho_{a} C}{m_{a}} \min \left(Q, Q_{a}\right),
$$

where $V$ is the cavity volume, $B_{0}$ is the magnetic field strength, $\rho_{a}$ is the local density of axions with energy corresponding to the cavity frequency, $Q$ is the loaded quality factor of the cavity, $Q_{a}$ is the ratio of the energy of the halo axions to their energy spread, equivalent to a "quality factor" for the halo axion signal, and $C$ is a mode dependent form factor which is largest for the fundamental transverse magnetic mode, $T M_{010}$. The quantity $C$ is given by

$$
C=\frac{\left|\int_{V} d^{3} x \mathbf{E}_{\omega} \cdot \mathbf{B}_{\mathbf{0}}\right|^{2}}{B_{0}^{2} V \int_{V} d^{3} x \epsilon\left|\mathbf{E}_{\omega}\right|^{2}},
$$

in which $\mathbf{E}_{\omega}(\mathbf{x}) e^{i \omega t}$ is the time dependent electric field of the mode under consideration, $\mathbf{B}_{\mathbf{0}}(\mathbf{x})$ is the static magnetic field in the cavity and $\epsilon$ is the dielectric constant of the medium inside the cavity. The frequency-dependent form factor is evaluated numerically. Eq. (3) can be recast in the convenient form

$$
\begin{aligned}
P= & 0.5 \times 10^{-21} \mathrm{~W}\left(\frac{V}{500 \mathrm{~L}}\right)\left(\frac{B_{0}}{7 \mathrm{~T}}\right)^{2} C\left(\frac{g_{\gamma}}{0.36}\right)^{2} \\
& \times\left(\frac{\rho_{a}}{0.5 \times 10^{-24} \mathrm{~g} \cdot \mathrm{cm}^{-3}}\right)\left(\frac{\nu_{a}}{1 \mathrm{GHz}}\right)\left(\frac{\min \left[Q, Q_{a}\right]}{10^{5}}\right),
\end{aligned}
$$

where $\nu_{a}$ is the axion mass frequency. As the experiment operates with the cavity at critical coupling, half the power developed in the cavity is lost to its walls and half is passed to the receiver chain.

The HR channel became fully operational in August, 2002. A schematic of ADMX, showing both the MR and HR channels, is given in Fig. [1 A more detailed illustration of the magnet, cavity and cryogenic components is shown in Fig. 2

The microwave cavity has an inner volume, $V$, of $189 \mathrm{~L}$. The frequency of the $T M_{010}$ mode can be tuned by moving a pair of metal or dielectric rods inside the cavity. The cavity is located in the bore of a superconducting solenoid, which generates a static magnetic field, $B_{0}$, of $7.8 \mathrm{~T}$. The voltage developed across a probe coupled to the electromagnetic field inside the cavity is passed to the receiver chain. During typical operation, $Q$ is approximately $7 \times 10^{4}$ and the total noise temperature for the experiment, $T_{n}$, is conservatively estimated to be $3.7 \mathrm{~K}$, including contributions from both the cavity and the receiver chain.

The first segment of the receiver chain is common to both the MR and HR channels. It consists of a cryogenic GaAs HFET amplifier built by NRAO, a crystal bandpass filter and mixers. At the end of this segment, 


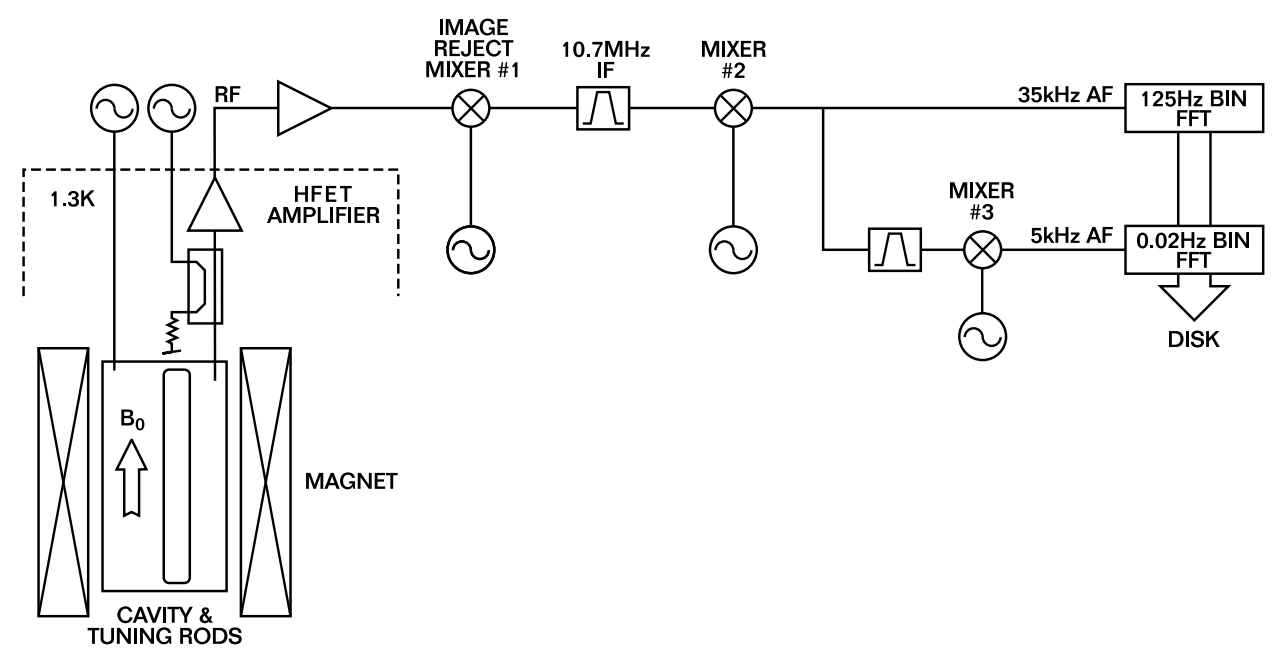

FIG. 1: Schematic diagram of the receiver chain.

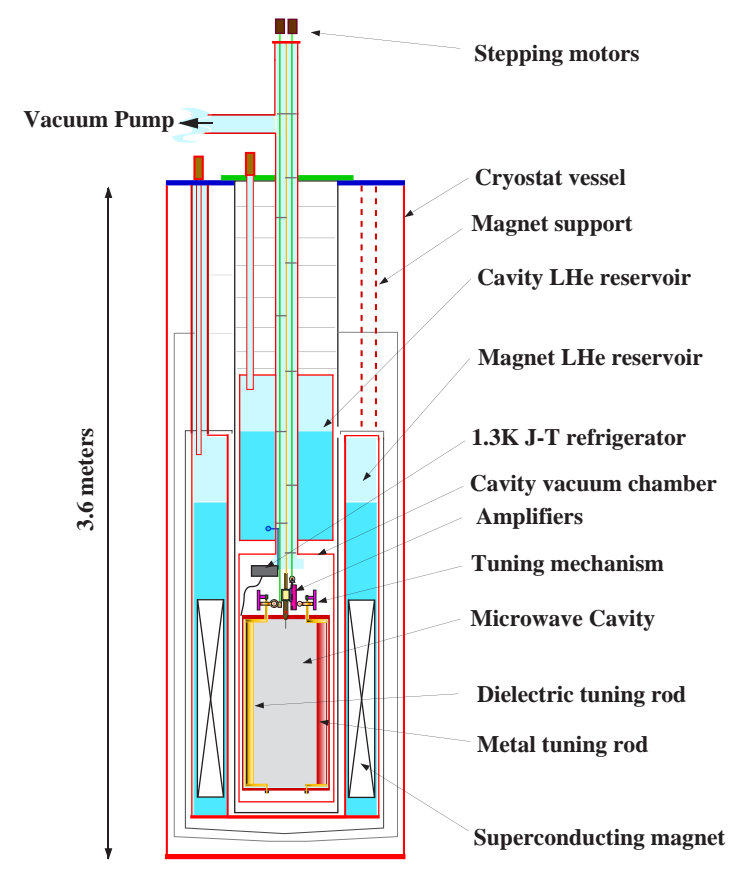

FIG. 2: Sketch of the ADMX detector.

the signal is centered at $35 \mathrm{kHz}$, with a $50 \mathrm{kHz}$ span. The MR signal is sampled directly after this part of the receiver chain. The HR channel contains an additional bandpass filter and mixer, resulting in a spectrum centered at $5 \mathrm{kHz}$ with a $6 \mathrm{kHz}$ span.

Time traces of the voltage output from the HR channel, consisting of $2^{20}$ data points, are taken with a sampling frequency of $20 \mathrm{kHz}$. This results in a data stream of $52.4 \mathrm{~s}$ in length, corresponding to $0.019 \mathrm{~Hz}$ resolution in the frequency spectrum. The data were primarily taken in parallel with the operations of the MR channel over a period beginning in November, 2002 and ending May, 2004. Continuous HR coverage has been obtained and candidate peak elimination performed for the frequency range 478-525 MHz. Data with $Q$ less than 40000 and/or cavity temperature above $5 \mathrm{~K}$ were discarded. In these cases, additional data were taken to ensure coverage of the entire range.

\section{HIGH RESOLUTION ANALYSIS}

We use the HR channel to search for narrow peaks caused by flows of cold axions through the detector. The background is thermal and electronic noise plus narrow lines from the rf environment of the experiment. These environmental peaks are signals from nearby emitters (e.g. computer clocks) which leak into the cavity by a variety of means.

When placing limits on cold flows of axions, we assume that the flows are steady, i.e. the rates of change of velocity, velocity dispersion and density of the flows are slow compared to the time scale of the experiment. The assumption of a steady flow implies that the signal we are searching for is always present. Even so, the kinetic energy term in Eq.(2) and the corresponding signal frequency change over time due to the Earth's rotational and orbital motions. In addition to a signal frequency shift in data taken at different times, apparent broadening of the signal occurs because its frequency shifts while the data are being taken.

In this section, we first describe the signal frequency shifts due to the Earth's rotation and orbital motion, and the associated signal broadening (subsection A). Next, we describe the properties of the noise in the HR channel (subsection B). In subsection C, we describe how we correct each spectrum for systematic effects introduced by the receiver chain. Finally, in subsection D, we describe how candidate peaks are selected and shown not to be caused by cold flows of dark matter axions. 


\section{A. Axion signal properties}

An axion signal will undergo diurnal and annual modulations due to the Earth's rotation and orbital motion, respectively [20]. Thus, the frequency at which axions are resonantly converted to photons will shift. We show here that this frequency modulation cannot move a signal by an amount which is larger than the detector bandwidth. As the energy of an axion in the ADMX detector is nonrelativistic (Eq. (2)), the shift in frequency of the signal, $\Delta f$, due to a change in velocity of the axion flow relative to the detector, $\Delta v$, is

$$
\Delta f=\frac{f v \Delta v}{c^{2}} .
$$

We have investigated the magnitude of both annual and diurnal signal modulation at $f=500 \mathrm{MHz}$. The velocity of a dark matter flow relative to the Earth will be in the range $100-1000 \mathrm{~km} / \mathrm{s}$. We chose $600 \mathrm{~km} / \mathrm{s}$ as a representative value for the purpose of estimation. For the daily modulation, we have assumed that the detector is located at the Earth's equator and that, in the frame in which the axis of rotation of the Earth is stationary, the flow velocity is first aligned and then anti-aligned (or vice versa) with the detector's motion due to the Earth's rotation over the course of a day. These assumptions result in the largest possible change in relative velocity between the detector and the flow due to the Earth's rotation. The Earth's rotational velocity is $0.4 \mathrm{~km} / \mathrm{s}$ at the equator. The resulting daily signal modulation is of order $1 \mathrm{~Hz}$. For the annual modulation, to again maximize the change in relative velocity, we have also considered the case of extreme flow velocity alignment with the Earth's orbital motion. The Earth's orbital velocity is $30 \mathrm{~km} / \mathrm{s}$. The annual modulation produces the larger frequency shift, of order $100 \mathrm{~Hz}$ within a year. The HR channel has a $6 \mathrm{kHz}$ bandwidth, ensuring that an axion peak will appear in spectra taken with center frequency equal to a previously observed axion signal frequency.

The signal broadening, $\delta f$, due to a change $\delta v$ in flow velocity while data are being taken is

$$
\delta f=\frac{f v \delta v}{c^{2}} .
$$

The most significant signal broadening is due to the Earth's rotation. Using the same assumptions as before, we find that the broadening is at most $4 \times 10^{-3} \mathrm{~Hz}$ during the $52 \mathrm{~s}$ taken to acquire a single time trace. This is less than the spectral resolution of $0.019 \mathrm{~Hz}$. The broadening due to the Earth's orbital motion is only of order $10^{-4} \mathrm{~Hz}$ in this same time interval.

As the signal broadening due to the Earth's rotation and orbital motion is negligible, we can use Eq. (7) to relate the width, $\delta f$, of a signal peak to the velocity dispersion, $\delta v$, of the axion flow that causes it. In general, we do not know the velocity dispersion of the cold axion flows which we search for, although we note that 14. claims an upper limit of $53 \mathrm{~m} / \mathrm{s}$ on the velocity dispersion of the Big Flow. Subsequently, we do not know the signal width. To compensate, we perform our search at multiple resolutions by combining $0.019 \mathrm{~Hz}$ wide bins. These searches are referred to as $n$-bin searches, where $n=1,2,4,8,64,512$ and 4096. For $f=500 \mathrm{MHz}$ and $v=300 \mathrm{~km} / \mathrm{s}$, the corresponding flow velocity dispersions are

$$
\delta v_{n}=12 n \mathrm{~m} / \mathrm{s}\left(\frac{300 \mathrm{~km} / \mathrm{s}}{v}\right) .
$$

Further details on the $n$-bin searhces are given in Section IIID.

\section{B. Noise in the HR Channel}

The power output from the HR channel is expressed in units of $\sigma$, the rms noise power. This noise power is related to the noise temperature, $T_{n}$, via

$$
\sigma=k_{B} T_{n} \sqrt{\frac{b}{t}}
$$

where $k_{B}$ is Boltzmann's constant, $b$ is the frequency resolution and $t$ is the acquisition time. The total noise temperature $T_{n}=T_{C}+T_{e l}$, where $T_{C}$ is the physical cavity temperature and $T_{e l}$ is the electronic noise contribution from the receiver chain. As no averaging is performed in HR sampling, $b=1 / t$. Thus, the rms noise power is

$$
\sigma=k_{B} b T_{n} .
$$

Output power is normalized to $\sigma$ and $T_{n}$ is used to determine this power. We verified Eq. (10) experimentally by allowing the cavity to warm and observing that $\sigma$ is proportional to $T_{C}$. As this is our calibration of the power output from the cavity, it is important that we understand the noise in the HR channel.

The noise in the HR channel is observed to have an exponential distribution. We now explain why this is expected. The noise in a single bin is the sum of independent sine and cosine components, as no averaging occurs. We expect that the noise amplitude, $a$, for a single component (i.e. sine or cosine) has a Gaussian probability distribution,

$$
\frac{d P}{d a}=\frac{1}{\sqrt{2 \pi} \sigma_{a}} \exp \left(-\frac{a^{2}}{2 \sigma_{a}^{2}}\right)
$$

where $\sigma_{a}$ is the standard deviation. Indeed, the energy distribution should be proportional to a Boltzmann factor, $\exp (-E / k T)$, and non-relativistic and classical energies, such as $E=m v^{2} / 2$ or $E=k x^{2} / 2$ are proportional to squares of the amplitude.

As there are two components per bin, the addition of $n$ bins is that of $2 n$ independent contributions. The sum of $2 n$ independent normal-distributed components is described by a chi-square distribution with $2 n$ degrees of 
freedom. Thus, the probability distribution for an $n$-bin is a $\chi^{2}(2 n)$ distribution. We demonstrate this explicitly in the following.

The probability distribution, $d P / d p_{n}$, of observing noise power $p_{n}$ in an $n$-bin is

$\frac{d P}{d p_{n}}=\left(\prod_{i=1}^{2 n} \int_{-\infty}^{\infty} d a_{i}\right) \frac{\exp \left(-\frac{1}{2 \sigma_{a}^{2}} \sum_{j=1}^{2 n} a_{j}^{2}\right)}{\left(\sqrt{2 \pi} \sigma_{a}\right)^{2 n}} \delta\left(p_{n}-\sum_{k=1}^{2 n} \frac{a_{k}^{2}}{2}\right)$

Evaluating the above expression,

$$
\frac{d P}{d p_{n}}=\frac{p_{n}{ }^{n-1}}{(n-1) ! \sigma_{a}^{2 n}} \exp \left(-\frac{p_{n}}{\sigma_{a}^{2}}\right)
$$

For $n=1$,

$$
\frac{d P}{d p_{1}}=\frac{1}{\sigma_{a}^{2}} \exp \left(-\frac{p_{1}}{\sigma_{a}^{2}}\right)
$$

which is indeed a simple exponential, as expected.

Using this noise distribution, we can easily see that the average (rms) noise power in the one bin search, $\sigma$, is $\sigma=\sigma_{a}^{2}$. Substituting this in Eq. (14), the noise power distribution function becomes

$$
\frac{d P}{d p_{1}}=\frac{1}{\sigma} \exp \left(-\frac{p_{1}}{\sigma}\right) .
$$

For each individual spectrum, the baseline noise level, $\sigma$, is determined by plotting the number of frequency bins, $N_{p}$, with power between $p$ and $p+\Delta p$ against $p$. According to Eq. (15),

$$
N_{p}=\frac{N \Delta p}{\sigma} \exp \left(-\frac{p}{\sigma}\right)
$$

where $N$ is the total number of data points. As

$$
\ln N_{p}=-\frac{p}{\sigma}+\ln \left(\frac{N \Delta p}{\sigma}\right)
$$

$\sigma$ is the inverse of the slope of the $\ln N_{p}$ versus $p$ plot. Fig. 3] demonstrates that the data is in good agreement with this relation for $p$ less than $20 \sigma$. The deviation of the data from Eq. (17) for $p$ greater than $20 \sigma$ is due to the fact that our background is not pure noise, but also contains environmental signals of a non-statistical nature.

As we combine an increasing number of bins, the noise power probability distribution approaches a Gaussian, in accordance with the central limit theorem. The righthand side of Eq. (13) approaches a Gaussian in the limit of large $n$. We have examined a large sample of noise in each $n$-bin search and verified that it is distributed according to Eq. (13). Figure 4 illustrates statistics for the 8-bin search and Fig. 5 shows statistics for the 4096bin search, a near Gaussian curve. Figures 3 through 5 illustrates the progression from exponential to Gaussian noise power probability distribution.

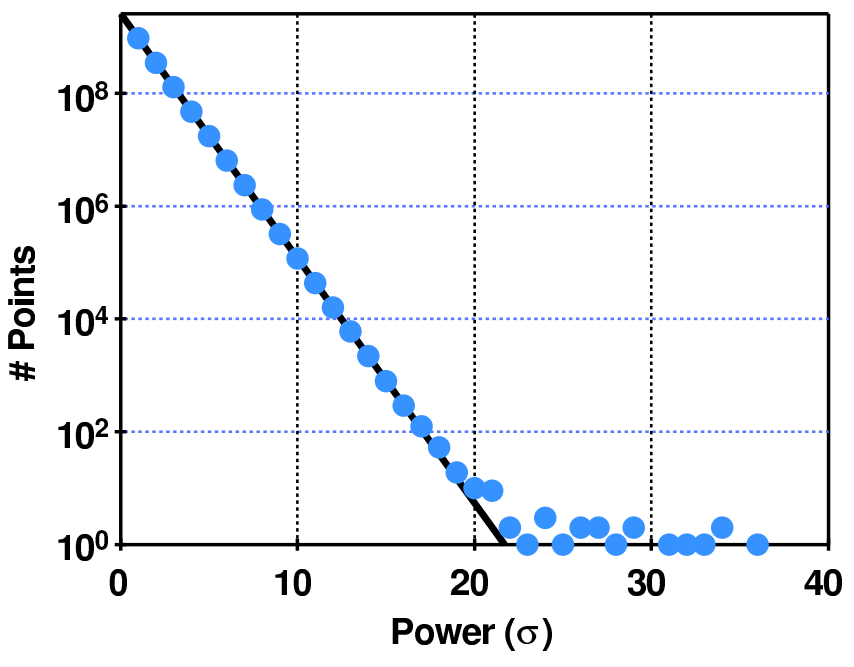

FIG. 3: Power distribution for a large sample of 1-bin data.

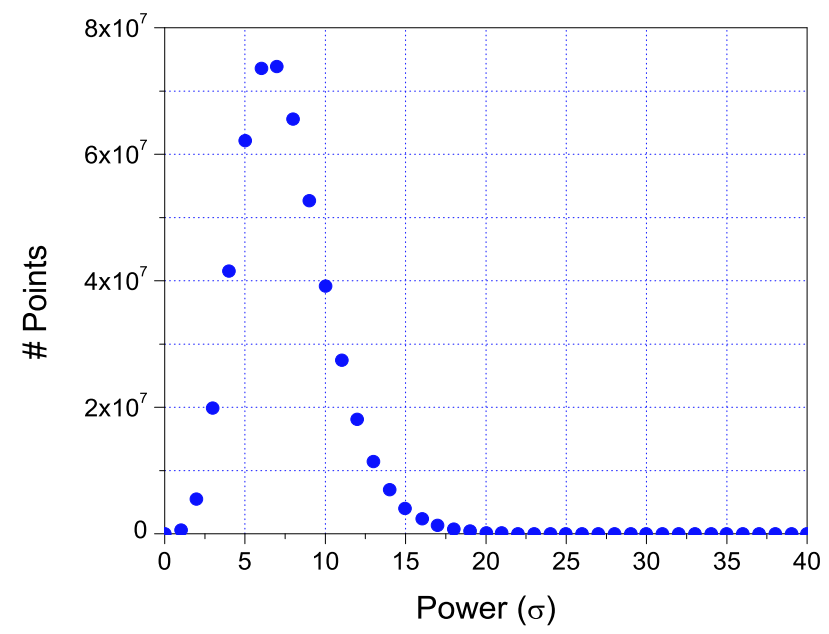

FIG. 4: Power distribution for a large sample of 8-bin data.

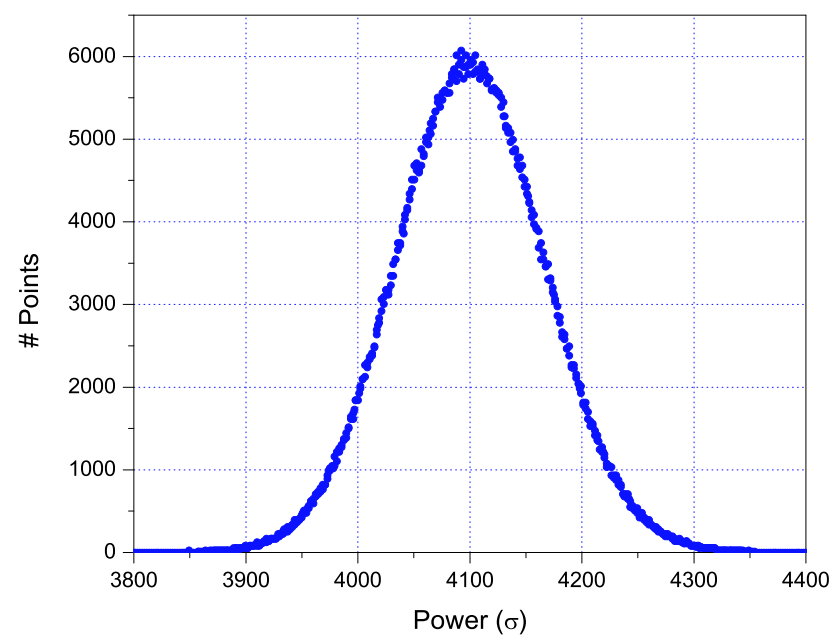

FIG. 5: Power distribution for a large sample of 4096-bin data. 


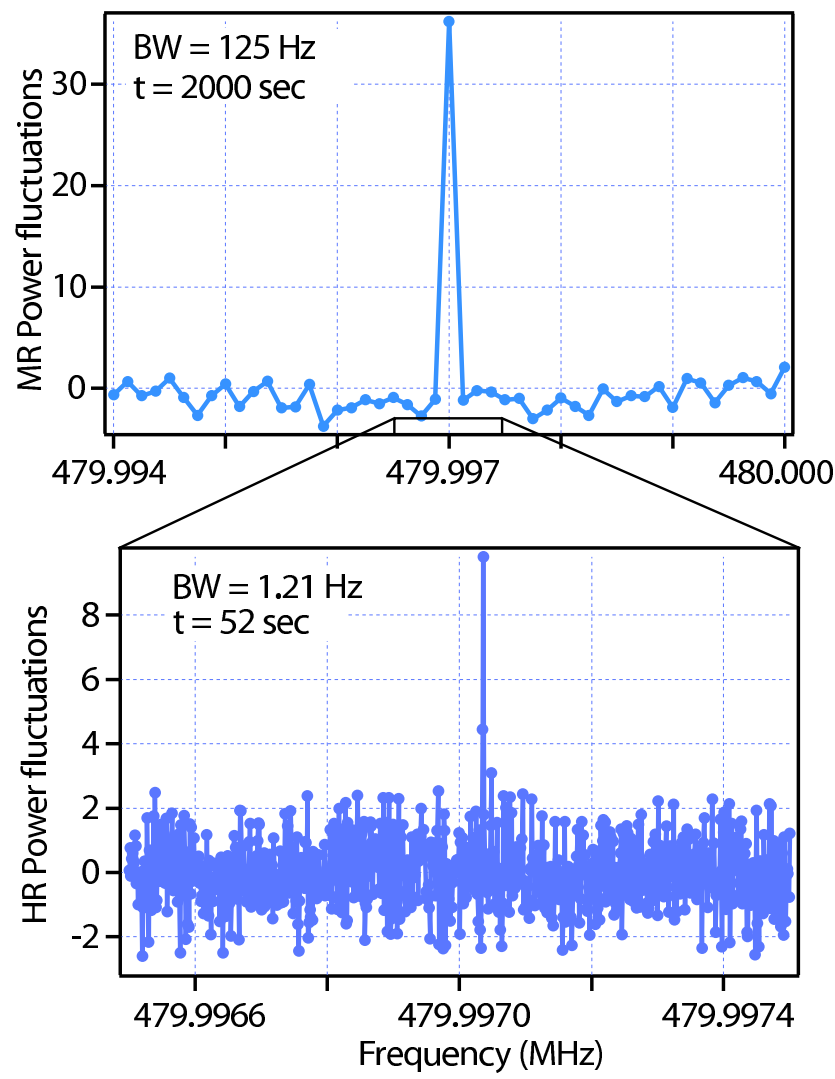

FIG. 6: An environmental peak as it appears in the MR search (top) and the 64-bin HR search. The unit for the vertical axis is the rms power fluctuation in each case.

In addition to examining the behavior of the noise statistics, we have performed a cross-calibration between the HR and MR channels. The signal power of an environmental peak, observed at $480 \mathrm{MHz}$ and shown in Fig. [6] was examined in both the HR and MR channels. The observed HR signal power was $(1.8 \pm 0.1) \times 10^{-22} \mathrm{~W}$, where the error quoted is the statistical uncertainty. The MR channel observed signal power $1.7 \times 10^{-22} \mathrm{~W}$, in agreement with the HR channel. Note that the MR signal was acquired with a much longer integration time than that of the HR signal (2000 s for MR versus $52 \mathrm{~s}$ for HR).

The combination of the calibration of the noise power with cavity temperature, the consistency between expected and observed noise statistics, and the agreement of signal power observed in both the HR and MR channels, makes us confident that the signal power is accurately determined in the HR channel.

\section{Removal of systematic effects}

There are two systematic effects introduced in the receiver chain shown in Fig. 1] Two passband filters are present on the HR receiver chain: one with bandwidth $35 \mathrm{kHz}$ on the shared MR-HR section and a passive LC

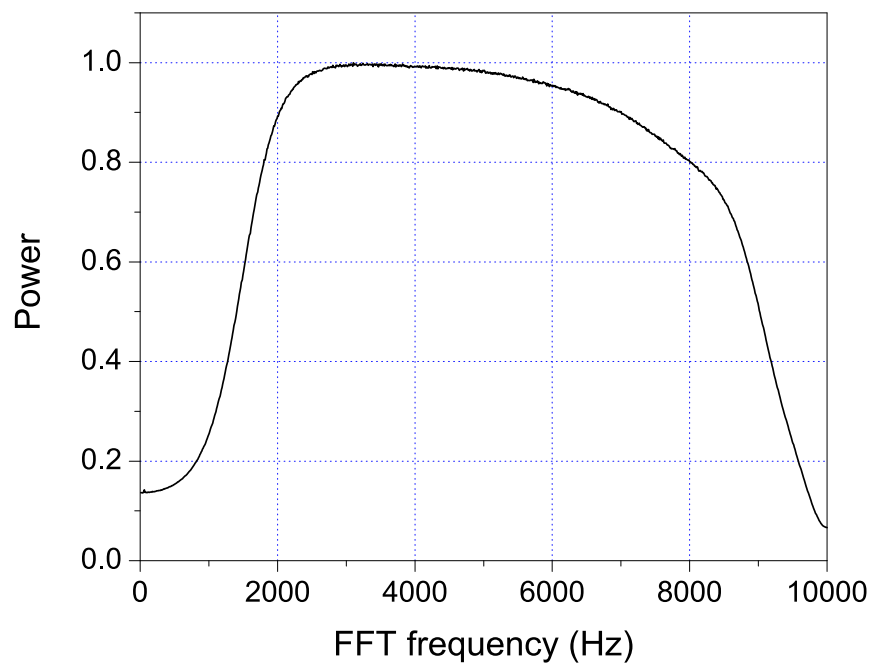

FIG. 7: HR filter response calibration data (512 bin average). The power has been normalized to the maximum power output.

filter of bandwidth $6 \mathrm{kHz}$, seen by the HR channel only. The combined response of both these filters has been analyzed and removed from the data. The second systematic effect is due to the frequency-dependent response of the coupling between the cavity and the first cryogenic amplifier. This effect is removed using the equivalent circuit model described later.

The combined passband filter response was determined by taking data with a white noise source at the rf input of the receiver chain. A total of 872 time traces were recorded over a two day period. In order to achieve a reasonably smooth calibration curve, 512 bins in the frequency spectrum for each time trace were averaged giving $9.77 \mathrm{~Hz}$ resolution. The combined average of all data is shown in Fig. 7 This measured response was removed from all data used in the HR search, as follows. The raw power spectra have frequency $0-10 \mathrm{kHz}$, where the center frequency of $5 \mathrm{kHz}$ has been mixed down from the cavity frequency. Each raw power spectrum is cropped to the region $2-8 \mathrm{kHz}$ to remove the frequencies not within the LC filter bandwidth. Each remaining frequency bin is then weighted by a factor equal to the receiver chain response at the given frequency divided by the maximum receiver chain response. Interpolation for frequency points not specifically included in the calibration curve is performed by assuming that each point on the calibration curve was representative of 512 bins centered on that frequency, so all power corresponding to frequencies within that range is normalized by the same factor. As the calibration curve varies slowly with frequency within the window to which each spectra is cropped, this is an adequate treatment of the normalization.

In the MR channel, the effect of the cavity-amplifier coupling is described using an equivalent-circuit model 21]. This model has been adapted for use in the HR channel. The frequency dependent response of the cavity 


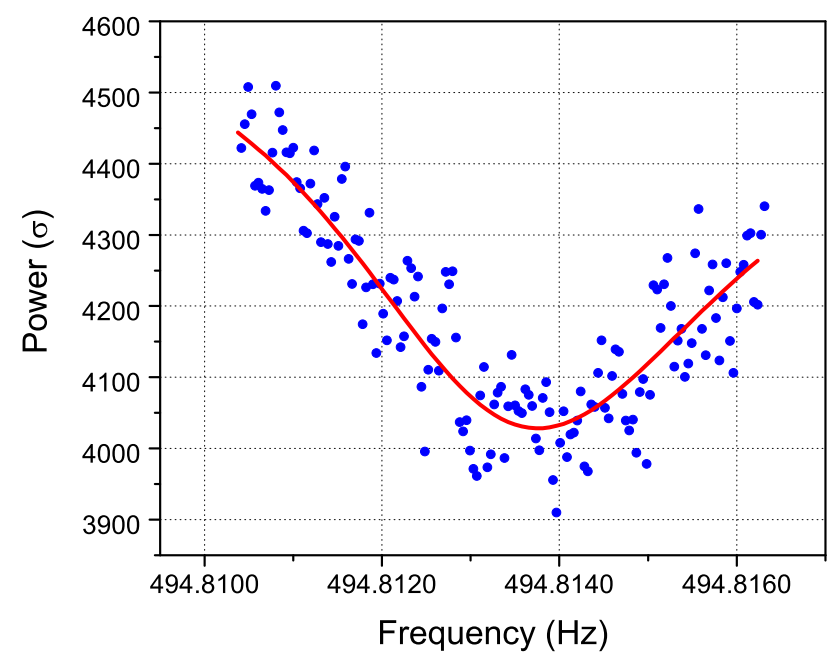

FIG. 8: Sample 4096-bin spectrum before correction for the cavity-amplifier coupling. The line is the fit obtained using the equivalent circuit model.

amplifier coupling is most evident in the 4096-bin search, thus this is the data used to apply the equivalent circuit model. A sample spectrum before correction is shown in Fig. 8

In the equivalent-circuit model, each frequency is given by $\Delta$, the number of bins it is offset from the bin of the center frequency, measured in units of the 4096-bin resolution, i.e. $b_{4096}=78.1 \mathrm{~Hz}$. The equivalent-circuit model predicts that the power (in units of the rms noise) at the NRAO amplifier output (the point labelled "RF" in Fig. (1) in the 4096-bin search at the frequency offset $\Delta$ is

$$
P(\Delta)=\frac{a_{1}+8 a_{3}\left(\frac{\Delta-a_{5}}{a_{2}}\right)^{2}+4 a_{4}\left(\frac{\Delta-a_{5}}{a_{2}}\right)}{1+4\left(\frac{\Delta-a_{5}}{a_{2}}\right)^{2}},
$$

where the parameters $a_{1}$ through $a_{5}$ are

$$
\begin{aligned}
& a_{1}=\left(b_{4096} / b\right)\left(T_{C}+T_{I}+T_{V}\right) / T_{n} \\
& a_{2}=f_{0} /\left(b_{4096} Q\right) \\
& a_{3}=\left(b_{4096} / b\right)\left(T_{I}+T_{V}+\left(T_{I}-T_{V}\right) \cos (2 k L)\right) / T_{n}(21) \\
& a_{4}=\left(b_{4096} / b\right)\left(\left(T_{I}-T_{V}\right) \sin (2 k L)\right) / T_{n} \text { and } \\
& a_{5}=\left(f_{0}-f_{c e n}\right) / b_{4096}
\end{aligned}
$$

In the above expressions, $T_{C}$ is the physical temperature of the microwave cavity, $T_{I}$ and $T_{V}$ are the current and voltage noise, respectively, contributed by the amplifier, $T_{n}$ is the noise temperature contributed from all components, $b$ is the frequency resolution of the HR channel, i.e. $0.019 \mathrm{~Hz}, L$ is the electrical (cable) length from the cavity to the HFET amplifer, $f_{0}$ is the cavity resonant frequency, $f_{c e n}$ is the center frequency of the spectrum and $k$ is the wavenumber corresponding to frequency $f_{c e n}+b \Delta$. The factor $b_{4096} / b$ appears in the parameters $a_{1}, a_{3}$ and $a_{4}$ as it is an overall factor which results from normalizing the power to the single bin noise baseline. In practice, the parameters $a_{1}$ through $a_{5}$ are established by fitting. The line in Fig. 8 shows the fit obtained using the equivalent circuit model.

Large peaks in the data, e.g. an axion signal or environmental peak, are removed before fitting to prevent bias. The 4096-bin spectrum is used to perform the fit and then the original 1-bin spectrum is corrected to remove the systematic effect. The weighting factors are calculated using Eq. (18) and the fitted parameters, $a_{1}$ through $a_{5}$, at the center of each bin of width $b_{4096}$. These factors are the ratio of the fit at a given point to the maximum value of the fit. Each 1-bin is multiplied by the factor calculated for the bin of width $b_{4096}$ within which it falls.

The removal of the cavity-amplifier coupling and the passband filter response using the techniques described above has been demonstrated to result in flat HR spectra.

\section{Axion signal search procedure}

We now describe the search for an axion signal and summarize the analysis performed on each time trace.

The width of an axion signal is determined by the signal frequency, axion velocity and flow velocity dispersion (Eq. (7)), the latter being the most uncertain variable. $n$-bin searches, where $n$ is the number of adjacent 1-bins added together ( $n=1,2,4,8,64,512$ and 4096), are conducted to allow for various velocity dispersions. For searches with $n>1$, there is an overlap between successive $n$-bins such that each $n$-bin overlaps with the last half of the previous and first half of the following $n$-bin. This scheme is illustrated for the 2, 4 and 8-bin searches in Fig. 9

The search for an axion signal is performed by scanning each spectrum for peaks above a certain threshold. All such peaks are considered candidate axion signals. The thresholds are set at a level where there is only a small probability that a pure noise peak will occur and such that the number of frequencies considered as candidate axion peaks is manageable. The candidate thresholds used were $20,25,30,40,120,650$ and $4500 \sigma$, in increasing order of $n$.

All time traces are analyzed in the same manner. A fast Fourier transform is performed and an initial estimate of $\sigma$ is obtained by fitting the 1-bin noise distribution to Eq. (17). Systematic effects are then removed, i.e. the corrections described in Section IIIC for the filter passband response and cavity-amplifier coupling are performed. "Large" peaks not included in the equivalent circuit model fit for the cavity-amplifier response are defined to be those greater than $120 \%$ of the search threshold for each $n$-bin search. After the removal of systematic effects, the 1-bin noise distribution is again fitted to Eq. (17) to obtain the true value of $\sigma$ and the search for peaks above the thresholds takes place.

The axion mass is not known, requiring that a range 


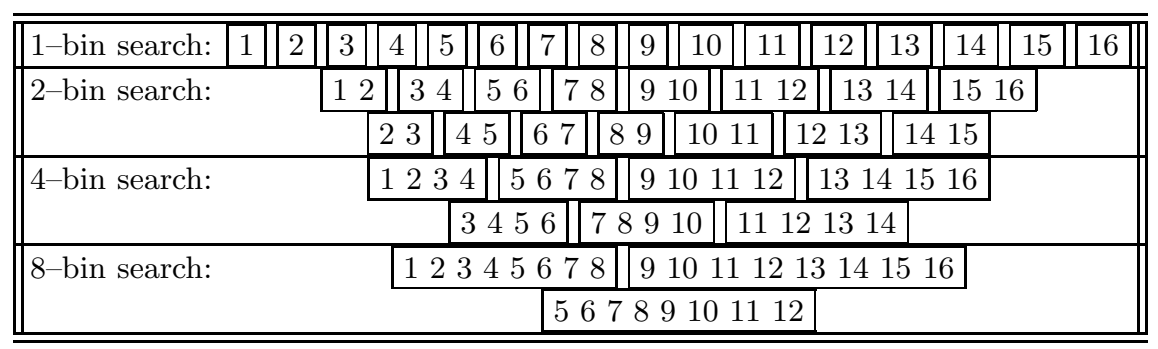

FIG. 9: Illustration of the addition scheme for the 2, 4 and 8-bin searches. The numbers correspond to the data points of the 1-bin search. Numbers within the same box are bins added together to form a single datum in the $n$-bin searches with $n>1$.

of frequencies must be examined. Full HR coverage has been obtained for the region $478-525 \mathrm{MHz}$, corresponding to axion masses between 1.98 and $2.17 \mu \mathrm{eV}$. The selected frequency range is examined in three stages for axion peaks, as follows:

Stage 1: Data for the entire selected frequency range is taken. The frequency step between successive spectra is approximately $1 \mathrm{kHz}$, i.e. the center frequency of each spectrum differs from the previous spectrum by $1 \mathrm{kHz}$. Frequencies at which candidate axion peaks occur are recorded for further examination during stage 2.

Stage 2: Multiple time traces are taken at each candidate frequency from stage 1 . The steady flow assumption described in Section IIA means that a peak will appear in spectra taken with center frequency equal to the candidate frequency from stage 1 if such a peak is an axion signal. The frequencies of persistent peaks, i.e. peaks that appear during both stage 1 and 2 are examined further in stage 3.

Stage 3: Frequencies of persistent peaks undergo a three-part examination. The first step is to repeat stage 2 , to ensure the peaks still persist. Secondly, the warm port attenuator is removed from the cavity and multiple time traces taken. If the peak is due to external radio signals entering the cavity (an environmental peak), the signal power will increase dramatically. If the signal originates in the cavity due to axion-photon conversion, the power developed in the cavity will remain the same as that for the normal configuration. The third step is to use an external antenna probe as a further confirmation that the signal is environmental. Some difficulties were encountered with the antenna probe, due to polarization of environmental signals. However, the second step is adequate to confirm that peaks are environmental. If a persistent peak is determined to not be environmental, a final test will confirm that it is an axion signal. The power in such a signal must grow proportionally with the square of the magnetic field ( $B_{0}$ in Eq. (5) $)$ and disappear when the magnetic field is switched off.

No axion peaks were found in the range $478-525 \mathrm{MHz}$ using this approach. The exclusion limit calculated from this data is discussed in the following section.

\section{RESULTS}

Over the frequency range $478-525 \mathrm{MHz}$, we derive an upper limit on the density of individual flows of axion dark matter as a function of the velocity dispersion of the flow. The corresponding axion mass range is 1.97$2.17 \mu \mathrm{eV}$. Each $n$-bin search places an upper limit on the density of a flow with maximum velocity dispersion, $\delta v_{n}$, as given by Eq. (8).

Several factors reduce the power developed in an axion peak from that given in Eq. (5). The experiment is operated near critical coupling of the cavity to the preamplifier, so that half this power is observed when the cavity resonance frequency, $f_{0}$, is precisely tuned to the axion energy. If $f_{0}$ is not at the center of a 1-bin, the power is spread into adjacent bins, as discussed below. When the axion energy is off-resonance, but still within the cavity bandwidth at a frequency $f$, the Lorentzian cavity response reduces the power developed by an additional factor of

$$
h(f)=\frac{1}{1+4 Q^{2}\left(\frac{f}{f_{0}}-1\right)^{2}} .
$$

To be conservative, we calculate the limits at points where successive spectra overlap, i.e. at the frequency offset from $f_{0}$ that minimizes $h(f)$.

If a narrow axion peak falls at the center of a 1-bin, all power is deposited in that 1-bin. However, if such a peak does not fall at the center of a 1-bin, the power will be spread over several 1-bins. We now calculate the minimum power in a single $n$-bin caused by a randomly situated, infinitely narrow axion line. The data recorded is the voltage output from the cavity as a function of time. The voltage as a function of frequency is obtained by Fourier transformation and then squared to obtain a raw "power" spectrum. The actual power is obtained by comparison to the rms noise power. The data are sampled for a finite amount of time and thus, the Fourier transformation of the output, $\mathcal{F}(f)$, will be of the voltage multiplied by a windowing function, i.e.

$$
\mathcal{F}(f)=\int_{-\infty}^{\infty} v(t) w(t) \exp (i 2 \pi f t) d t
$$

where $v(t)$ is the measured output voltage and $w(t)$ is 
the windowing function for a sampling period $T$,

$$
w(t)=\left\{\begin{array}{l}
1 \text { if }-T / 2 \leq t \leq T / 2, \\
0 \text { otherwise }
\end{array}\right.
$$

Eq. (25) is equivalent to

$$
\mathcal{F}(f)=\int_{-\infty}^{\infty} V(k) W(f-k) d k,
$$

where $V(f)$ and $W(f)$ are the Fourier transforms of the output voltage, $v(t)$, and the windowing function, $w(t)$, i.e. $\mathcal{F}(f)$ is the convolution of $V(f)$ and $W(f)$, given by

$$
W(f)=\frac{\sin (\pi f T)}{\pi f} .
$$

Discretizing Eq. (27) and inserting Eq. (28), we have

$$
\mathcal{F}(f)=\sum_{m=0}^{N} V\left(\left(m+\frac{1}{2}\right) b\right) \frac{\sin \left(\pi\left(\frac{f}{b}-\left(m+\frac{1}{2}\right)\right)\right)}{\pi\left(\frac{f}{b}-\left(m+\frac{1}{2}\right)\right)},
$$

where $b$ is the frequency resolution of the HR channel, $2 N$ points are taken in the original time trace, and the center frequency of the $j$ th 1 -bin is $(j+1 / 2) b$. Thus, for an axion signal of frequency $f$ falling in 1-bin $j$, a fraction of the power

$$
g(m)=\left(\frac{\sin (m \pi+\delta)}{m \pi+\delta}\right)^{2}
$$

is lost to the $m$ th 1-bin from 1-bin $j$, where $\delta=\pi(m+$ $1 / 2-f / b)$. If $\delta=0$, i.e the axion signal frequency is exactly equal to a 1-bin center frequency, all the power is deposited in a single 1-bin. However, if this is not the case, power is lost to other 1-bins. In setting limits, we assume that the power loss is maximal.

The maximum power loss occurs when a signal in the 1-bin search falls exactly between the center frequency of two adjacent 1-bins. In this case, when $\delta=\pi / 2$, Eq. (30) shows that $40.5 \%$ of the power will be deposited in each of two 1-bins. In $n$-bin seaches with $n \geq 2$, not as much power is lost to other $n$-bins, due to the overlap between successive $n$-bins. The minimum power deposited in an $n$-bin is $81 \%$ for $n=2,87 \%$ for $n=4$ and $93 \%$ for $n=8$. For $n=64,512$ and 4096, the amount of power not deposited in a single $n$-bin is negligible.

For the $n$-bin searches with $n=64,512$ and 4096, a background noise subtraction was performed which will lead to exclusion limits at the $97.7 \%$ confidence level. These limits are derived using the power at which the sum of the signal power and background noise power have a $97.7 \%$ probability to exceed the candidate thresholds. We call this power the "effective" threshold for each search. The effective thresholds are obtained by integrating the noise probability distribution, Eq. (13), numerically solving for the background noise power corresponding to the $97.7 \%$ confidence level for each $n$ and subtracting these values from the original candidate thresholds.
TABLE I: Effective power thresholds for all $n$-bin searches, with the frequency resolutions, $b_{n}$ and corresponding maximum flow velocity dispersions, $\delta v_{n}$, for a flow velocity of $600 \mathrm{~km} / \mathrm{s}$.

\begin{tabular}{rrrr}
\hline \hline$n$ & $\begin{array}{r}\text { Effective } \\
\text { threshold }(\sigma)\end{array}$ & $\begin{array}{c}b_{n} \\
(\mathrm{~Hz})\end{array}$ & $\begin{array}{r}\delta v_{n} \\
(\mathrm{~m} / \mathrm{s})\end{array}$ \\
\hline 1 & 20 & 0.019 & 6 \\
2 & 25 & 0.038 & 10 \\
4 & 30 & 0.076 & 20 \\
8 & 40 & 0.15 & 50 \\
64 & 71 & 1.2 & 400 \\
512 & 182 & 9.8 & 3000 \\
4096 & 531 & 78 & 20000 \\
\hline \hline
\end{tabular}

TABLE II: Numerically calculated values of the form factor, $C$, and amplifier noise temperatures, $T_{e l}$, from NRAO specifications.

\begin{tabular}{ccc}
\hline \hline Frequency $(\mathrm{MHz})$ & $\mathrm{C}$ & $T_{e l}(\mathrm{~K})$ \\
\hline 450 & 0.43 & 1.9 \\
475 & 0.42 & 1.9 \\
500 & 0.41 & 1.9 \\
520 & 0.38 & 1.9 \\
550 & 0.36 & 2.0 \\
\hline \hline
\end{tabular}

For $n=64,512$ and 4096, the effective thresholds are 71, 182 and $531 \sigma$, respectively. For smaller values of $n$, background noise subtraction does not significantly improve the limits and the effective threshold was taken to be the candidate threshold. Table \ summarizes this information and shows the frequency resolution of each search with the corresponding maximum flow velocity dispersion from Eq. (8) for $v=600 \mathrm{~km} / \mathrm{s}$.

Our exclusion limits were calculated for an axion signal with power above the effective threshold reduced by the appropriate factors. These factors arise from the critical coupling, the Lorentzian cavity response and the maximum power loss due to the peak not falling in the center of an $n$-bin, as outlined above. Equations (5) and (10) were used, for both KSVZ and DFSZ axion couplings. The cavity volume, $V$, is $189 \mathrm{~L}$. Measured values of the quality factor, $Q$, the magnetic field, $B_{0}$, and the cavity temperature, $T_{C}$, are recorded in each data file. $\mathrm{Nu}$ merically determined values of the form factor, $C$ are given in Table The electronic noise temperature, $T_{e l}$, was conservatively taken from the specifications of the NRAO amplifier, the dominant source of noise in the receiver chain, although our measurements indicate that $T_{e l}$ is less than specified. These values are also given in Table III Linear interpolation between values at the frequencies specified was used to obtain values of $C$ and $T_{e l}$ at all frequencies.

The 2-bin search density exclusion limit obtained using these values is shown in Fig. [10 For values of $n$ other than $n=2$, the exclusion limits differ by only constant factors. The constant factors are 1.60, 1.00, 1.12, 1.39, 2.53, 5.90 and 17.2 for $n=1,2,4,8,64,512$ and 4096, 


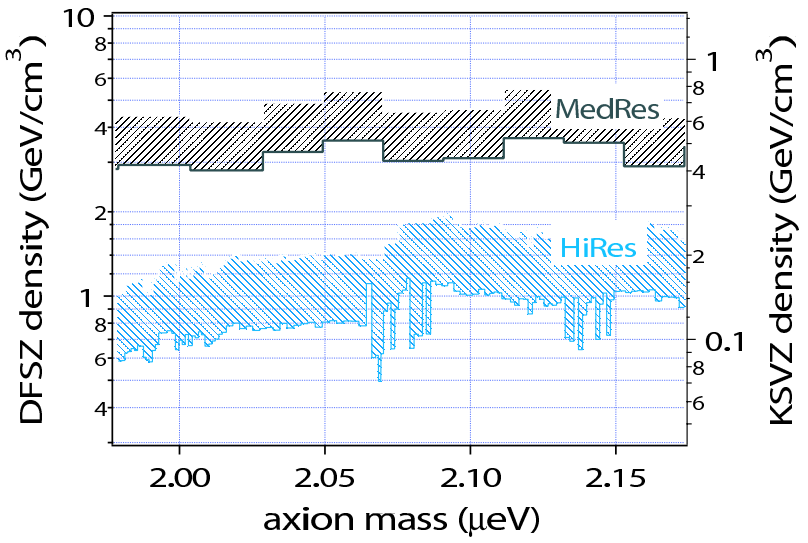

FIG. 10: 97.7\% confidence level limits for the HR 2-bin search on the density of any local axion dark matter flow as a function of axion mass, for the DFSZ and KSVZ $a \gamma \gamma$ coupling strengths. Also shown is the previous ADMX limit using the MR channel. The HR limits assume that the flow velocity dispersion is less than $\delta v_{2}$ given by Eq. (8).

respectively.

\section{DISCUSSION}

We have obtained exclusion limits on the density in local flows of cold axions over a wide range of velocity dispersions. The most stringent limit, shown in Fig. 10] is from the 2-bin search. For a flow velocity of $600 \mathrm{~km} / \mathrm{s}$ relative to the detector, the 2 -bin search corresponds to a maximum flow velocity dispersion of $10 \mathrm{~m} / \mathrm{s}$. The 1bin search limit is less general, in that the corresponding flow velocity dispersion is half that of the 2-bin limit. It is also less stringent; much more power may be lost due to a signal occurring away from the center of a bin than in the $n=2$ case. For $n>2$, the limits are more general, but the larger power threshold of the searches make them less stringent.

The largest flow predicted by the caustic ring model has density $1.7 \times 10^{-24} \mathrm{~g} / \mathrm{cm}^{3}\left(0.95 \mathrm{GeV} / \mathrm{cm}^{3}\right)$, velocity of approximately $300 \mathrm{~km} / \mathrm{s}$ relative to the detector, and velocity dispersion less than $53 \mathrm{~m} / \mathrm{s}$ [14]. Using Eq. (7) with Table \ and the information displayed in Fig. 10 multiplied by the appropriate factors of 1.12 to obtain the 4-bin limit, it can be seen that the 4-bin search, corresponding to maximum velocity $50 \mathrm{~m} / \mathrm{s}$ for $v=300$ $\mathrm{km} / \mathrm{s}$, would detect this flow if it consisted of KSVZ axions. For DFSZ axions, this flow would be detected for approximately half the search range.

Figure 10 demonstrates that the high resolution analysis improves the detection capabilities of ADMX when a significant fraction of the local dark matter density is due to flows from the incomplete thermalization of matter that has only recently fallen onto the halo. The addition of this channel to ADMX provides an improvement of a factor of 3 over our previous medium resolution analysis.

\section{Acknowledgements}

This research is supported in part by the U.S. Department of Energy under Contract W-7405-ENG-48 at Lawrence Livermore National Laboratory, under grant DE-FG02-97ER41029 at the University of Florida, and by an IBM Einstein Endowed Fellowship at the Institute for Advanced Study.
[1] C. L. Bennett et al., Astrophys. J. Suppl. 148, 1 (2003).

[2] R. D. Peccei and H. R. Quinn, Phys. Rev. D 16, 1791 (1977); R. D. Peccei and H. R. Quinn, Phys. Rev. Lett. 38, 1440 (1977); S. Weinberg, Phys. Rev. Lett. 40, 223 (1978); F. Wilczek, Phys. Rev. Lett. 40, 279 (1978).

[3] L. F. Abbott and P. Sikivie, Phys. Lett. B 120, 133 (1983). J. Preskill, M. B. Wise and F. Wilczek, Phys. Lett. B 120, 127 (1983). M. Dine and W. Fischler, Phys. Lett. B 120, 137 (1983).

[4] S. Asztalos et al., Phys. Rev. D64 092003 (2001).

[5] P. Sikivie, Phys. Rev. Lett. 51, 1415 (1983) [Erratumibid. 52, 695 (1984)].

[6] J. F. Navarro, C. S. Frenk and S. D. M. White, Astrophys. J. 462, 563 (1996); B. Moore, F. Governato, T. Quinn, J. Stadel and G. Lake, Astrophys. J. 499, L5 (1998).

[7] P. Sikivie, Phys. Lett. B432 139 (1998).

[8] P. Sikivie, Phys. Rev. D 60, 063501 (1999).

[9] D. Lynden-Bell, Mon. Not. Roy. Astron. Soc. 136, 101 (1967).
[10] S. J. Asztalos et al., Phys. Rev. D 69, 011101(R) (2004).

[11] K. Freese, P. Gondolo, H. J. Newberg and M. Lewis, Phys. Rev. Lett. 92, 111301 (2004); K. Freese, P. Gondolo and H. J. Newberg, Phys. Rev. D 71, 043516 (2005).

[12] P. Sikivie and J. Ipser, Phys. Lett. B291 288 (1992); A. Natarajan and P. Sikivie, Phys. Rev. D 72, 083513 (2005).

[13] J.E. Kim, Phys. Rep. 1501 (1987); H.-Y. Cheng, Phys. Rep. 1581 (1988); M.S. Turner, Phys. Rep. 19767 (1990); G.G. Raffelt, Phys. Rep. 1981 (1990).

[14] P. Sikivie, Phys. Lett. B 567, 1 (2003).

[15] E. I. Gates, G. Gyuk and M. S. Turner, Astrophys. J. 449, L123 (1995).

[16] L. Duffy et al., Phys. Rev. Lett. 95, 091304 (2005).

[17] J. E. Kim, Phys. Rev. Lett. 43, 103 (1979); M. A. Shifman, A. I. Vainshtein and V. I. Zakharov, Nucl. Phys. B 166, 493 (1980).

[18] M. Dine, W. Fischler and M. Srednicki, Phys. Lett. B 104, 199 (1981). A. R. Zhitnitsky, Sov. J. Nucl. Phys. 31, 260 (1980) [Yad. Fiz. 31, 497 (1980)]. 
[19] S. De Panfilis et al., Phys. Rev. Lett. 59, 839 (1987); C. Hagmann, P. Sikivie, N. S. Sullivan and D. B. Tanner, Phys. Rev. D 42, R1297 (1990); C. Hagmann et al., Phys. Rev. Lett. 80, 2043 (1998); H. Peng et al., Nucl. Instrum. Meth. A 444, 569 (2000); R. Bradley et al., Rev. Mod. Phys. 75, 777 (2003).
[20] M. S. Turner, Phys. Rev. D 42, 3572 (1990); F. S. Ling, P. Sikivie and S. Wick, Phys. Rev. D 70, 123503 (2004).

[21] E. J. Daw, PhD thesis, Massachusetts Institute of Technology, 1998. 